\title{
ROTATION RATE OF HIGH-LATITUDE SUNSPOTS
}

\author{
FRANCES TANG \\ Hale Observatories, ${ }^{*}$ California Institute of Technology, Pasadena, Calif. 91125, U.S.A.
}

(Received 12 February; in revised form 2 May, 1980)

\begin{abstract}
Rotation rate of 19 high latitude $\left(28-44^{\circ}\right)$ short-lived sunspots collected in 1978-1979 are compared with Newton and Nunn's (1951) recurrent spots rate. To reduce the effect of proper motion in spots of new regions, our measurements start only when the spots have matured or very nearly so. Compared with the expression $\omega=14.38-2.96 \sin ^{2} \phi$ derived from 1934-44 data by Newton and Nunn, our results show a slightly lower differential rotation in the $28-40^{\circ}$ zone. They are in better agreement with the Greenwich average results of the five solar cycles beginning 1878: $\omega=14.37-2.60 \sin ^{2} \phi$.
\end{abstract}

\section{Introduction}

The solar rotation rate for spots derived from recurrent sunspots in the solar cycle 1934-44 by Newton and Nunn (1951) has long been a classic. The sidereal rotation rate for recurrent spots was found to be $14.38-2.96 \sin ^{2} \phi$, where $\phi$ is the latitude. They showed that this result is in satisfactory accordance with values from similar data for the five previous cycles beginning 1878, a century ago. The same result was also shown recently by Kearns (1979) to be in agreement with his analysis of the 1978 data. While Newton and Nunn's analysis was plausible in many aspects and the extent of the data vast, it is lacking in one area, namely, the number of data points used in the high latitude zone.

Of the 136 recurrent spots used in the Newton and Nunn analysis, only two were in the above $30^{\circ}$ latitude in the solar cycle 1934-44. In the first four of the five previous cycles, there were no recurrent spots above $30^{\circ}$. The fifth cycle also had only two recurrent spots in the $30-35^{\circ}$ latitude zone. And so these very few spots played a very large role in shaping the tail-end of the much-quoted Newton and Nunn rotation curve, actually for recurrent spots, but popularly known simply as the Sun's rotation rate.

The difficulty, of course, lies beyond the investigator's control. The Sun simply does not produce many recurrent spots in its high latitudes.

The appearance of high latitude spots ushers in a new solar cycle but the high latitude is not a fertile land for spots. Only a very small fraction of spots manage to survive there. And the very high latitudes (the 40's) are reserved for none but the solar maximum year of the largest solar cycles. Kopecký $(1958,1959)$ compiled a list of all observed spot groups and spots with latitudes $\geq 40^{\circ}$ from 1874 to 1957 . There were 66 entries on the list, including those observed only once. The single most productive year of these very high latitudes spots was 1957 with 19 spots.

* Hale Observatories are operated jointly by the Carnegie Institution of Washington and the California Institute of Technology. 
A total of 29 spots ranging from $40-48^{\circ}$ were observed from 1955 to 1957 , the maximum years of the largest solar cycle on record. The rotation rate of a sunspot at $\mathrm{N} 48.3^{\circ}$ observed for an incredible 15 days in 1956 was reported by Waldmeier (1957) to be $12.35^{\circ} \pm 0.15^{\circ} \mathrm{d}^{-1}$, within $3 \%$ (slower) of the Newton and Nunn rate.

In his 1959 paper Kopecký had rotation rates of 7 single spots from the list of 66 with $40^{\circ}$ latitude and above that had a time lapse of 5 days or longer between observations. The data will be compared with ours in Section 4.

Since another solar maximum is upon us, we decided to collect and measure the shorter-lived spots in the high latitudes and to compare their rotation rates with those of the recurrent spots of the lower latitudes and to supplement Kopecký's very high latitude data.

\section{Data}

Since Big Bear Solar Observatory has been in full operation for less than a solar cycle, our study is confined to the current cycle which began in the latter part of 1975 . Full-disk filtergrams from the $22 \mathrm{~cm}$ vacuum refractor telescope with a $0.7 \AA$ FWFM band Fabry-Perot filter are used. The telescope optics are carried by invar rods which keep the optics rigidly located relative to one another and the magnification fixed. The Sun's disk is photographed on the film for $10 \mathrm{hr}$ at the rate of three per minute on a normal day. For our measurements, the $35 \mathrm{~mm}$ filtergram is projected in a Theodolite viewer onto a screen forming an image $18 \mathrm{~cm}$ in diameter. $X$ and $Y$ coordinates of the center of mass of the spot are read off from cross hairs and converted to heliographic longitude and latitude. Two independent measurements are made for each spot. Days when spots are within $20^{\circ}$ from the limbs are not used. Rotation rates are obtained from the difference in longitudes on the first and last day of measurements and the time lapse.

Data used in our study include those that satisfy all of the following conditions:

(1) Only spots with latitudes higher than $28^{\circ}$ are included.

(2) To greatly reduce, if not eliminate, the effect of proper motion commonly occurring in new bipolar spots during its growth stage, our first day measurement of a spot takes place only when the spot has matured or very nearly so. The area of a spot is usually a good indication of its developent stage.

(3) Although we do not take daily measurements of the spots used in this analysis, we make sure that no substantial morphological change takes place in the intervening days between the first and last measurement.

(4) The first and last measurement of the same spot is at least 4 days apart.

This way we collected 19 spots (all in 1978 and 1979) out of a total of 168 spots counted from the Solar Geophysical Data from 1975 through May of 1979 that lived at least 2 days at latitudes $28^{\circ}$ and higher. Table I lists the spots used in this study. The 'Remarks' column indicates the visual judgement of the development stage of the region the spot is in as well as the polarity of the spot. 
TABLE I

The sunspots used in this study

\begin{tabular}{|c|c|c|c|c|}
\hline No. & Latitute & Dates & $\begin{array}{l}\text { Siedereal rotation } \\
\text { rate }\left(\mathrm{d}^{-1}\right)\end{array}$ & Remarks \\
\hline 1. & +29.8 & Feb. $14-24,1978$ & $13.59 \pm 0.04$ & $\beta P$, large spot, newish region \\
\hline 2. & -31.1 & Mar. $13-19,1978$ & $13.51 \pm 0.06$ & $\beta P$, newish region \\
\hline 3. & -34.5 & Mar. $13-19,1978$ & $13.47 \pm 0.08$ & $\beta F$, newish region \\
\hline 4. & -44.0 & Apr. $12-23,1978$ & $12.91 \pm 0.04$ & $\alpha P$, medium stable spot \\
\hline 5. & -29.5 & May $10-26,1978$ & $13.74 \pm 0.06$ & $\alpha P$, small spot, oldish region \\
\hline 6. & +44.0 & Aug. $15-19,1978$ & $14.14 \pm 0.07$ & $\beta P-\alpha P$, new region \\
\hline 7. & +31.5 & Sep. $7-15,1978$ & $13.97 \pm 0.07$ & $\begin{array}{l}\beta P, \text { developed region with new } \\
\text { flux emerging in between }\end{array}$ \\
\hline 8. & +33.7 & Sep. 8-13, 1978 & $13.77 \pm 0.04$ & $\begin{array}{l}\beta F, \text { developed region with new } \\
\text { flux emerging in between }\end{array}$ \\
\hline 9. & +38.0 & Sep. $12-22,1978$ & $13.32 \pm 0.06$ & $\beta B-\alpha P$, medium stable spot \\
\hline 10. & +28.8 & Sep. $13-23,1978$ & $13.85 \pm 0.04$ & $\beta P$, large spot, young region \\
\hline 11. & +28.1 & Oct. $21-27,1978$ & $13.88 \pm 0.07$ & $\beta P$, new region \\
\hline 12. & +31.8 & Oct. $21-27,1978$ & $13.87 \pm 0.03$ & $\alpha P$, small spot \\
\hline 13. & -28.1 & Nov. $17-26,1978$ & $13.62 \pm 0.04$ & $\alpha P$, stable spot, youngish region \\
\hline 14. & -38.1 & Feb. $10-18,1979$ & $13.76 \pm 0.03$ & $\begin{array}{l}\beta P-\alpha P, \text { new spot, } P \text { drifted } \\
\text { poleward }\end{array}$ \\
\hline 15. & +36.0 & Feb. 23-Mar. 1, 1979 & $13.20 \pm 0.06$ & Elongated $P$ spot \\
\hline 16. & +28.5 & May 9-18, 1979 & $13.92 \pm 0.04$ & $\alpha P$, stable spot \\
\hline 17. & -31.3 & May $14-18,1979$ & $13.73 \pm 0.03$ & $\beta F$, new region \\
\hline 18. & -29.1 & May $14-20,1979$ & $13.67 \pm 0.07$ & $\beta P$, new region \\
\hline 19. & -34.0 & May $22-29,1979$ & $13.36 \pm 0.03$ & $\alpha P$, stable small spot \\
\hline
\end{tabular}

\section{Results}

The results are superposed on Newton and Nunn's curve as crosses in Figure 1. The average uncertainty introduced into the rotation rate due to random error in measurements is $\pm 0.05^{\circ} \mathrm{d}^{-1}$. The circled crosses indicate the average results when binned in $28-30^{\circ}, 30-35^{\circ}$, and $35-40^{\circ}$ zones of latitude. Because of the scatter, an average is not given for the $40^{\circ}-45^{\circ}$ zone.

The following points are noted from our data:

(1) The scatter in our data is larger than that of Newton and Nunn, as one might suspect when dealing with the rotation rate of spots spanning an average of 7.4 days instead of 27 days.

(2) The scatter is not centered along the Newton and Nunn curve. It is shifted toward a higher rotation rate (less differential rotation).

(3) The averaged result agrees well with Newton and Nunn's curve at $28.8^{\circ}$ latitude. It is $1.2 \%$ higher in the $30-35^{\circ}$ zone and $1 \%$ higher in the $35-40^{\circ}$ zone (in which we have only three data points). Up to $40^{\circ}$ (the next zone $40-45^{\circ}$ will be discussed separately) the averaged results show a definite differential rotation but at a slightly flatter rate than Newton's and Nunn's. In the expression $\omega=A-B^{2} \sin \phi$, $B$ is found to be 2.96 for the solar cycle $1934-44$ by Newton and Nunn, in contrast to the average $B$ value of 2.60 from the previous five cycles. The broken line in Figure 1 


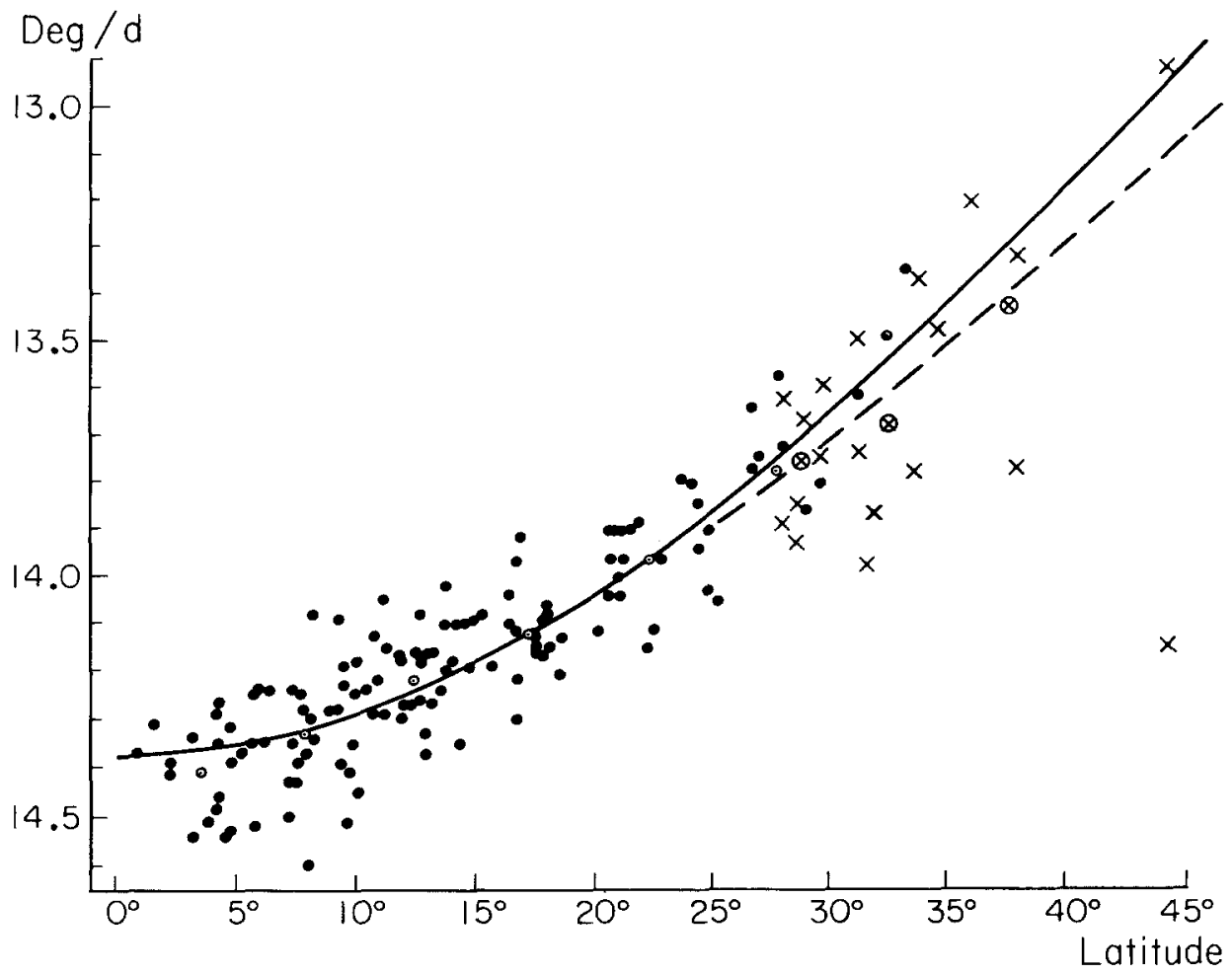

Fig. 1. Dots, circled dots and solid line up to $35^{\circ}$ latitude are original Newton and Nunn's results from recurrent sunspots, $1934-44$. The curve beyond $35^{\circ}$ latitude is contracted from the same expression $\omega=14.38-2.96 \sin ^{2} \phi$. Ordinate: daily sidereal motion, deg $\mathrm{d}^{-1}$. Abscissa: heliographic latitude. The broken line is constructed from the expression $\omega=14.37-2.60 \sin ^{2} \phi$, the average result of the 5 cycles beginning 1878. Crosses are data from our 1978-79 study. Circled crosses are binned results in 28-30 $30-35^{\circ}$, and $35-40^{\circ}$ latitude zones.

is constructed with the latter $B$ value. Our short-lived spots of $1978-79$ are closer to these values.

(4) For non-recurrent spots that lived 9-10 days, Newton and Nunn found them to rotate more differentially then the recurrent spots with a $B$ value of 3.36 . Our data show the opposite tendency.

(5) In the $40-45^{\circ}$ latitude zone we have only two spots. One at $\mathrm{S} 44^{\circ}$ lived 12 days and had a rotation rate of $12.91^{\circ} \mathrm{d}^{-1}$ comparable to the $12.95^{\circ} \mathrm{d}^{-1}$ rate calculated from Newton and Nunn's expression. The other spot at $\mathrm{N} 44^{\circ}$ had a highly unusual rotation rate. The region was born on August 12, 1978. Bipolar spots emerged the next day. By the 15 th, $P$ spot was at its maximum and we took our first measurement. Four days later it diminished to a tiny spot and we ended our measurement on the 19 th. The four days have an average rotation of $14.14^{\circ} \mathrm{d}^{-1}$. This is $9 \%$ higher than the rate calculated from Newton and Nunn's expression. 


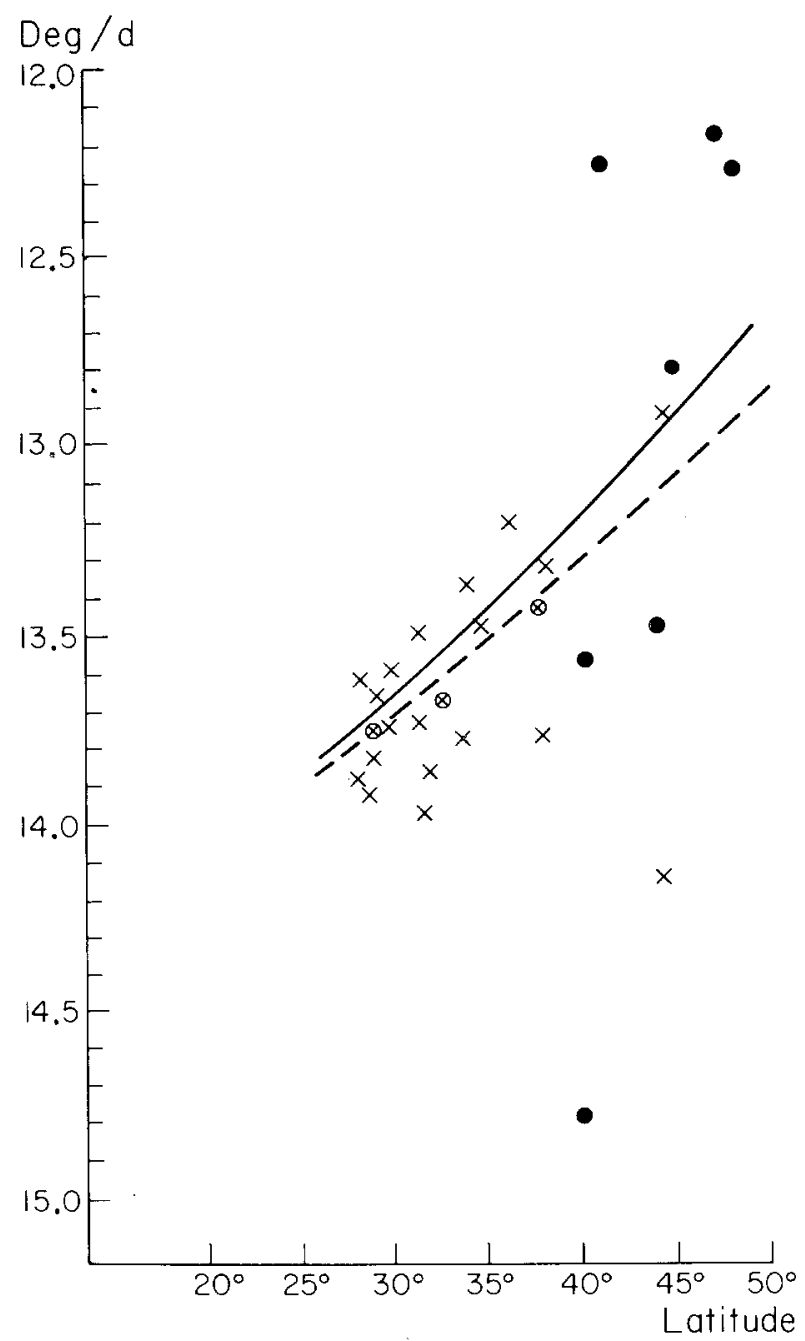

Fig. 2. 1978-79 spots are shown as crosses. Dots are 1956-57 spots from Kopecký. Solid line corresponds to $\omega=14.38-2.96 \sin ^{2} \phi$. Broken line corresponds to $\omega=14.37-2.60 \sin ^{2} \phi$.

\section{Discussion}

The seven data points from Kopecký's latitudes $40^{\circ}$ and above spots mentioned earlier in Section 1 are plotted as dots along our data (the crosses) in Figure 2. As pointed out in his paper, the recurrent rates of Newton and Nunn (solid line) and the Greenwich average (broken line) are reasonable approximations of these spots. The rotation rates of these spots also show a greater scatter than our 1978-79 data. We do not know if the greater scatter at increasing latitudes is real. There are differences in the treatment of data between the two sets. Kopecký's data, for instance, made no restriction on the maturity of the spot such as condition (2) imposed on our data in 
Section 2. One does not know the true effect of this restriction on the rotation rates until one knows the life story of high latitudes sunspots. Waldmeier (1957) analyzed the daily rotation rate of the spot at $\mathrm{N} 48.3^{\circ}$ for 15 days and found the second half of the disk passage had a near constant rate that was $0.57^{\circ} \mathrm{d}^{-1}$ faster than the near constant rate of the first half of the disk passage. If all high latitude spots were to behave in this manner, one would still have difficulty explaining the scatter which is greater by a factor of 2 .

\section{Acknowledgements}

I would like to thank Prof. H. Zirin for suggesting the study and for helpful comments and Drs R. Moore and G. Hurford for helpful comments and discussion, and Dr R. Howard for the Mount Wilson Doppler data. I am grateful to the referee for calling my attention to the work done by Kopecky $(1958,1959)$ that I was not aware of. This work was supported by NASA under grant NGL 05-002-034 and the NSF under grant ATM 6-21132.

\section{References}

Kearns, M.: 1979, Solar Phys. 62, 393.

Kopecký, M.: 1958, Bull. Astron. Inst. Czech. 9, 34.

Kopecký, M.: 1959, Bull. Astron. Inst. Czech. 10, 12.

Newton, H. W. and Nunn, M. L.: 1951, Monthly Notices Roy. Astron. Soc. 111, 413.

Waldmeier, M.: 1957, Z. Astrophys. 43, 29. 